\section{X-RAY ANALYSIS IN INDUSTRY}

X-RAY analysis provides a new tool for solving Industrial problems. It is used in many laboratories in Great Britain, and the researchers who have become expert in handling it are constantly developing new methods, and discovering new types of problem to which it can be successfully applied. The Conference arranged by the Institute of Physics, and held at Cambridge on April 10 and 11, had as its object the interchange of ideas and knowledge between the various groups of workers. Two previous attempts to call this Conference have been frus. trated, the first by the onset of war and the second by the difficult conditions of the summer of 1940. In order that the object which it had in view might at any rate be partially achieved, the Institute of Physics decided to publish the series of papers which appeared in the Journal of Scientific Instruments in May and July 1941 ; these papers formed the basis of discussions for the present Conference. Some anxiety was felt by those responsible for the arrangements, lest preoccupation with war-work would prevent many from attending, but the decision to proceed was made because the X-ray tool is being widely used for problems directly connected with the War. The large attendance at the Conference (some 280 participated) and the generally expressed appreciation of this opportunity for discussion have shown that this anxiety was unnecessary.

$\mathbf{X}$-ray analysis found its earliest application in the study of crystalline structure, the pattern of the atomic arrangement in the perfect crystal. The results of this analysis have had a profound influence on fundamental concepts of the nature of chemical combination. It has been realized that the law of combining proportions is not always the result of the grouping of atoms into molecules; in many classes of compound it represents the ratio of the numbers of atoms which are welded by the interatomic forces into one continuous pattern. It has been realized that one must be cautious in carrying over into the metallic field concepts based on organic and inorganic compounds, and the physical realities behind such terms as 'intermetallic compound', 'phase', and 'solid solution' have become much clearer. Such work ultimately influences technical applications, because it clarifies ideas.

The Conference concerned itself, however, with more direct applications of the $\mathrm{X}$-ray method, and the subject of crystal analysis was specifically excluded, or rather, its results were taken for granted. An actual crystalline material may be composed of fragments with different types of crystalline strueture. The crystallites have characteristics of size, of shape, of orientation, and of perfection, which may profoundly modify the macroscopic properties of the material. The perfect pattern is an ideal only attained by crystals under special conditions. In 'mixed crystals' various atoms may serve as proxies for each other, and an increasing number of crystals are proving to be tolerant to a remarkable extent of minor variations in their pattern without destroying its general plan. $\mathbf{X}$-ray analysis affords valuable information about their characteristics, and is often the only available method of attack; the study of such problems was the business of the Conference.

The progress of an X-ray analysis is reminiscent of that in a good detective story. It is an indirect method. We cannot extort a full confession from the guilty party. Instead, we are given a series of clues, which point to some possibilities and exclude others, until finally we have an accumulated weight of evidence which makes us confident we have arrived at the right solution. The indirect nature of the evidence is seen most clearly in the analysis of crystalline patterns. Theoretically, the observed $\mathbf{X}$-ray diffraction effects might be produced by an infinity of arrangements of scattering matter (in technical terms the phases of the scattered beams are not observable). A unique solution ' is only possible because other evidence can be called in support. The right solution must represent atoms of known number and type, with features of arrangement and spacing relative to each other which previous experience with other structures has shown to be characteristic. Instead of logically deducing a solution, intelligent guesses have to be made and tested against the observations. The same is true for $\mathbf{X}$-ray analysis of all types. The clues afforded by the more or less diffuse spots, arcs, or lines on an X-ray photograph of some mysterious material must be combined with a wealth of other knowledge if they are to be followed up. Much of the fascination of $\mathbf{X}$-ray analysis is due to its taking one into so many other fields of science. The X ray expert should not only understand the optical principles on which his science is founded; he must at the same time be a chemist, a metallurgist, a petrologist, or a biochemist. The optical principles can be logically deduced, but imagination, common-sense and a wide general knowledge lead him to make his intelligent guesses.

The wide range covered by the industrial applications is shown by the papers published last year. In the short time available at the Conference detailed discussion of all the papers was not practicable, and it was decided to group certain of the papers together and discuss them under the three headings : "Cameras for X-ray Analysis"; "The Application of X-ray Crystallography to Industrial Problems"; "X-ray Structure and Mechanical Properties". At the same time an exhibition of photographs, films and apparatus cognate to the above subjects was arranged. This exhibition was well supported, and examples of recent work and modern apparatus emphasized the very marked improvements in technique which have taken place in the last decade.

The papers, and the contributors to the discussion at the Conference itself, all came from laboratories in Great Britain. They did not represent a general review of the application of $\mathrm{X}$-ray analysis to industrial problems, which has been advanced by workers in many countries. The limitation was deliberate. The Conference was planned to be a comparing of notes and pooling of knowledge by the many $\mathrm{X}$-ray workers in Great Britain who desire to collaborate more closely, and it has done much to further this aim.

In each discussion the procedure adopted was that the main aspects of the subject were introduced by speakers who had contributed papers to the symposium, and afterwards a general discussion took place. This present account of the proceedings deals only with the more interesting items arising from the general discussions.

On cameras, two interesting developments may be mentioned, one by Orowan of the Cavendish Laboratory, Cambridge, describing the use of a rotating wire grid which enables much extra useful information to be obtained from rotating crystal photographs, and the other by Frommer of High Duty Alloys, 
Slough, concerning the design of very large cameras in which completed metallurgical products can be examined for measurement of internal stress.

The discussion on applications brought out the very great diversity of subjects now being investigated by X-ray methods. Bunn of Imperial Chemical Industries, Ltd. (Northwich), in his remarks emphasized the need for the production of an index of the known structures of chemical compounds, etc., as this would be a great help in the identification of compounds. This question was the subject of further comment by other speakers, and was referred to the organizing committee of the Conference with the view of promoting active steps to provide an index for X-ray workers.

The final discussion was concerned with the correlation of X-ray structure of metals and mechanical properties. Numerous speakers indicated how industry is developing this subject in connexion with such problems as the measurement of internal stresses and preferred orientation in strip and wire, and on the theoretical side Sir Lawrence Bragg showed how the measurements by Wood on the limiting crystallite size of metals can be used to estimate in a simple way their yield strength within very reasonable limits.

On April 10, Sir Lawrence Bragg delivered a lecture entitled "The History and Development of X-ray Analysis." This was a very enjoyable feature. It commenced with a fascinating account of the early work of himself and his father, the late Sir William Bragg, and after tracing the major developments up to the present time, ended with a statement of various problems, such as the structure of proteins, the 'fine' structure of deformed metal, ete., which were in his opinion on the threshold of solution.

As a result of the interest displayed at the Conference and the enthusiasm of the members, a resolution was passed suggesting that the organizing committee should take steps to set up a permanent organization under the ægis of the Institute of Physics to arrange similar conferences from time to time.

\section{CRYSTALLINITY IN CELLULOSE ESTERS}

$\mathrm{A}$ CCORDING to an article by W. O. Baker (Bell Lab. Rec., 20, No. 6, Feb., 1942) the toughness, strength and flexibility of plastics are influenced by the arrangement as well as the composition of their giant molecules. In an endeavour to discover the fundamental properties of certain compounds such as cellulose acetate and cellulose butyrate, which make these materials resistant to shock, bending, twisting and dimensional change, studies were undertaken on a molecular scale, the high magnification necessary being obtained by photographing $\mathrm{X}$-ray beams after passage through selected samples of the plastics. These photographs give patterns which can be measured to show molecular distances as small as a billionth of an inch, and they also indicate how the molecules are placed with respect to each other.

A minute cylindrical beam is passed through small flat sections about one millimetre thick, much of it expanding into coaxial cones on account of diffraction by the layers of molecules. After an exposure of three or four hours, circular records appear on the photographic film, the degree of crystallinity being indicated by the sharpness and number of circles recorded. In striking analogy to metals, it was found that the cellulose esters could be quenched by rapid cooling from the molten state. The long polymer molecules were then found to be disordered with respect to each other. Neighbours of a given molecule are quite randomly arranged although there is a tendency for sections of the chains to lie side by side. When cooled slowly, however, the molecules have a very ordered arrangement in local regions throughout the plastic. When they have the maximum disorder, the material tends to be most soft and flexible, and when they are most ordered, or crystallized, it is hardest and strongest, but sometimes brittle.

Two other methods of controlling the number of organized and disorganized molecules have long been used as working procedures in the technology of cellulose plastics. The first is to control the shape of the cellulose ester molecules by the amount of the reaction and the nature of the substituting groups so that they can only partially fit together, to give an ordered arrangement. The second, applying chiefly to lacquers such as aeroplane dopes and film formation, is the selection of a particular solvent which evaporates as the cellulose ester film dries. Various liquids were found to cause different amounts of molecular disorder in the resulting films.

The study showed that sections of the plastic's molecules could orient themselves in an ordered position in the solid state and that they undergo considerable torsional motion under the influence of temperature. Thus, it was possible to anneal quenched cellulose esters, and X-ray patterns show how this process causes the chain molecules to take up ordered positions. This ability of molecules to move in plastic solids even at ordinary temperatures appears to be closely related to their plasticity and capacity to bend and return to original form.

\section{DEVELOPMENTS IN ADULT EDUCATION*

\author{
By DR. BASIL A. YEAXLEE \\ University of Oxford
}

$\mathrm{D}^{\mathrm{s}}$ ESPITE all that has been done in the field of educational psychology adult education has still only an empirical basis. There has been practically no research in Great Britain and very little in the United States. Yet without such careful inquiry it is not possible to plan effectively the great extension of the movement that is desirable after the War or to develop the necessary variety of methods. The first stage is to determine the problems to which the attention of psychological investigators should be directed. The educational activities now so widespread among men and women of H.M. Forces should yield valuable data and suggestions.

It must be remembered that nothing like simple extension into this constituency of work on conventional lines was feasible. The opportunity was, and is, immense. But conditions as a rule prevent the systematic, continuous study and the maintenance of academic standards which characterize the courses and classes carried on among civilians in peace-time by university extra-mural departments and organizations such as the Workers' Educational Association. On the other hand, a very small percentage of the

* Substance of an address to the Education Section of the British Psychological Society at its Extended General Meeting, Brighton, April 10, 1942. 